\title{
GALAXY ORIENTATION IN SOME ABELL CLUSTERS
}

\author{
W. GODŁOWSKI AND F. BAIER \\ Obserwatorium Astronomiczne, Uniwersytet Jagielloński, \\ ul. Orla 171 30-244 Kraków, Poland \\ WIP Project Galaxienhaufen Universitat at Podsdam \\ An der Sternwarte 16 Podsdam Babelsberg Germany
}

We analyze a sample of galaxies in a region of three rich Abell Clusters. The data are taken from the Edinburgh Catalogue of the cluster. First, we divide the whole cluster area into different parts according to the assumed subclusters. Now we can find the position angles of the cluster and subclusters. We find strong evidence that the position angles of galaxies within our clusters are aligned to a large extent. For the cluster A754, position angles of galaxies tend to be perpendicular to the direction of the position angle of the cluster. Consequently, the angular momentum of galaxies are preferentially perpendicular to the cluster plane. For the cluster A14, position angles of galaxies tend to be parallel to the direction of the position angle of the cluster. Consequently, the angular momentum of galaxies are preferentially parallel to the cluster plane. For the cluster A3667 we obtain a more complicated picture suggesting that the alignment of galaxies in this cluster may have a different shape. From the distribution of the positions angles of galaxies we also found evidence for possible subclustering inside the whole cluster. This result is confirmed by the investigation of the distribution of the vectors normal to the galactic planes. Moreover we confirm the existence of a "line of sight" effect, originally found by Godłowski \& Ostrowski (1996) for galaxies belonging to the clusters in the Tully Catalogue (1988), for the clusters in our basic catalog.

\section{References}

Godłowski, W. 1994, MNRAS, 271, 19

Godłowski, W., Ostrowski, M. 1997, in press

Tully, R.B. 1988, Nearby Galaxies Catalog, Cambridge U. Press 